\title{
Isolation, identification and genomic characterization of the Asian lineage Zika virus imported to China
}

\author{
Yong-Qiang Deng ${ }^{1 \dagger}$, Hui Zhao ${ }^{1 \dagger}$, Xiao-Feng Li $^{1 \dagger}$, Na-Na Zhang ${ }^{2 \dagger}$, Zhong-Yu Liu ${ }^{1}$, Tao Jiang ${ }^{1}$, \\ Da-Yong Gu ${ }^{3}$, Lei Shi ${ }^{3}$, Jian-An $\mathrm{He}^{3}$, Hong-Jiang Wang ${ }^{1}$, Zhao-Zeng Sun ${ }^{4}$, Qing $\mathrm{Ye}^{1}$, \\ Dong-Yang Xie ${ }^{5}$, Wu-Chun Cao ${ }^{1 *}$ \& Cheng-Feng Qin ${ }^{1,2,5^{*}}$ \\ ${ }^{1}$ Department of Virology, State Key Laboratory of Pathogen and Biosecurity, Beijing Institute of Microbiology and Epidemiology, Beijing \\ 100071, China \\ ${ }^{2}$ Graduate School, Guangxi Medical University, Xining 530021, China \\ ${ }^{3}$ The Central Laboratory of Health Quarantine, Shenzhen Travel Healthcare Center, Shenzhen Entry-Exit Inspection and Quarantine Bureau, \\ Shenzhen 518033, China \\ ${ }^{4}$ Laboratory Animal Center, Academy of Military Medical Science, Beijing 100071, China \\ ${ }^{5}$ Graduate School, Anhui Medical University, Hefei 230032, China
}

Received March 9, 2016; accepted March 15, 2016; published online March 17, 2016

Citation: Deng, Y.Q., Zhao, H., Li, X.F., Zhang, N.N., Liu, Z.Y., Jiang, T., Gu, D.Y., Shi, L., He, J.A., Wang, H.J., Sun, Z.Z., Ye, Q., Xie, D.Y., Cao, W.C., and Qin, C.F. (2016). Isolation, identification and genomic characterization of the Asian lineage Zika virus imported to China. Sci China Life Sci 59, 428-430. doi: 10.1007/s11427-016-5043-4

Dear Editor,

Zika virus (ZIKV) used to be an unknown mosquito-borne flavivirus, and maintained its limited sylvatic circulation in a few African and Asian countries (Enfissi et al., 2016). Based on available clinical data, the symptoms in human infections with ZIKV are supposed to be similar to other arbovirus infections such as dengue, and characterized by fever, skin rashes, conjunctivitis, muscle and joint pain, malaise, and headache (Duffy et al., 2009). However, its occasional introduction to the Americas resulted in unexpected large outbreaks with millions of human cases since 2015 (Fauci and Morens, 2016). Even worsen, substantial evidence has linked microcephaly in infants, a severe form of birth defect, to ZIKV infection in pregnant women (Mlakar et al., 2016). On 1 February 2016, the World Health Organization has stated ZIKV and observed increase in neurological disorders and neonatal malformations a public health emergency of international concern. Now, ZIKV has rapidly spread to more than 52 countries and territories

†Contributed equally to this work

*Corresponding author (email: qincf@bmi.ac.cn; caowc@bmi.ac.cn) in Africa, Americas, Asia and the Pacific.

As of 6 March 2016, the National Health and Family Planning Commission of China has declared 10 imported ZIKV cases. On 19 February 2016, a 38-year-old Chinese male patient, returning from Samoa to Shenzhen, was finally laboratory confirmed as the third imported ZIKV case in mainland China. The patient had fever, conjunctival congestion and maculopapular rash, and recovered in a week. Here, we report the isolation, identification and genomic characterization of the first Chinese ZIKV strain (named SZ01) isolated from the imported case.

Two-day-old BALB/c suckling mice $(n=5)$ were inoculated intracranially with pretreated acute phase serum from the patient. Seven days post inoculation, obvious nervous system symptoms were seen in the majority of the inoculated animals, including inactivity, hind-leg paralysis and hypersomnia (Figure 1A). Then, the brains were dissected from the dying mice and grinded in DMEM medium (Life Technologies, USA) supplement with $2 \%$ fetal bovine serum (Life Technologies, USA), and total RNAs was extracted using QIAamp Viral RNA Mini Kit (Qiagen, Germany) according to the manufacturer's instructions. ZIKV- 


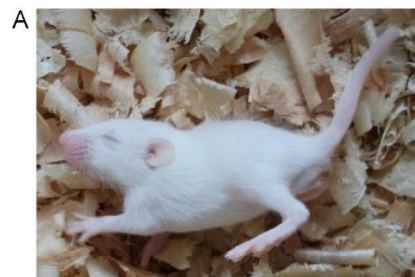

D
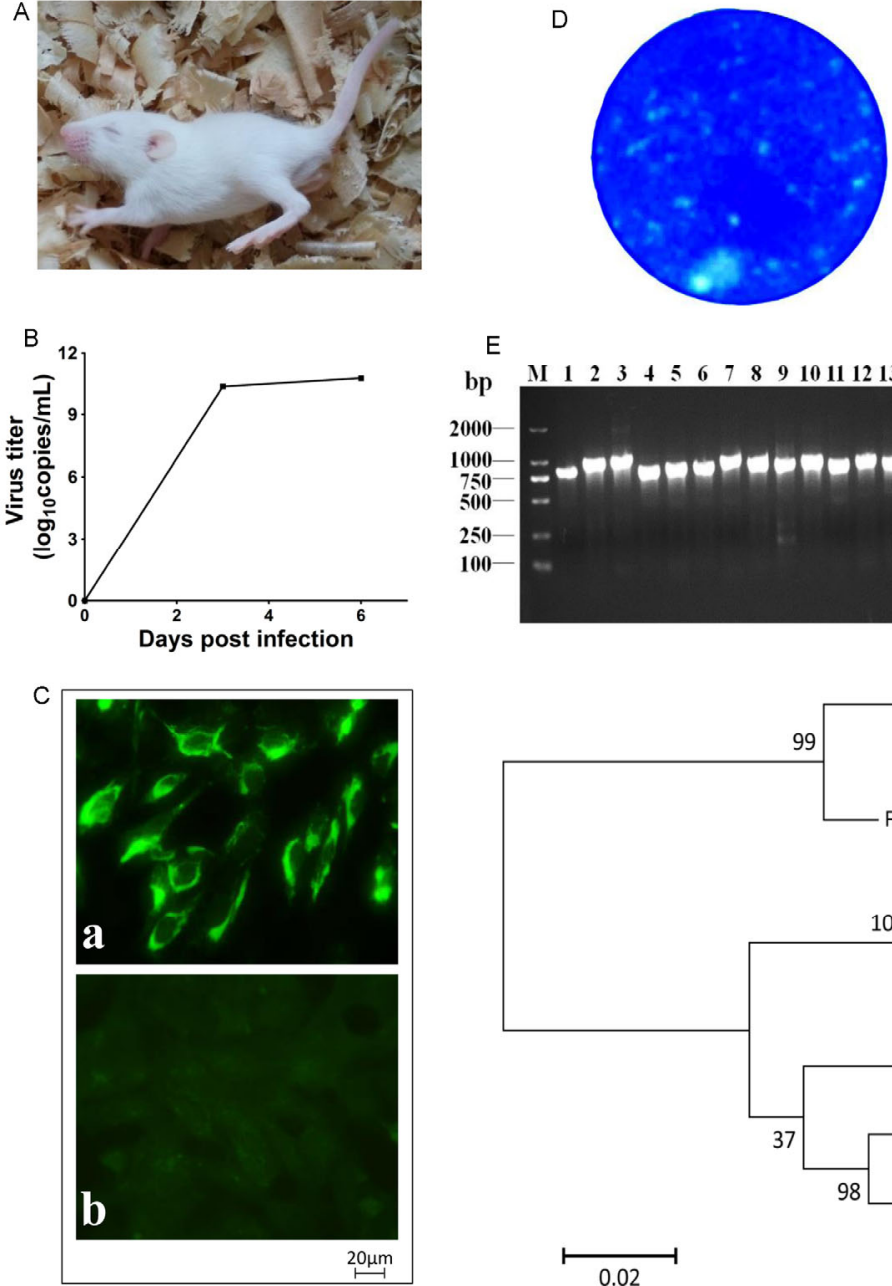

E

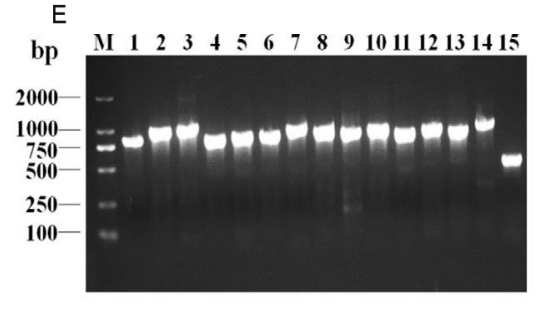

99

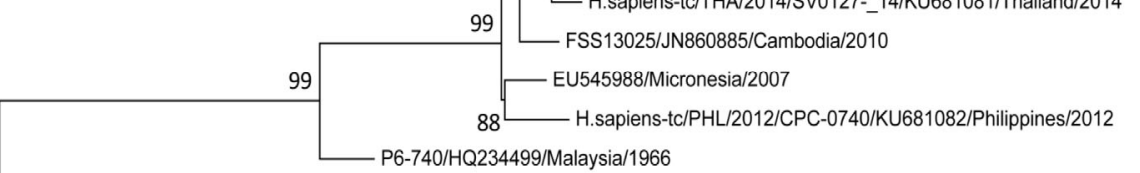

P6-740/HQ234499/Malaysia/1966

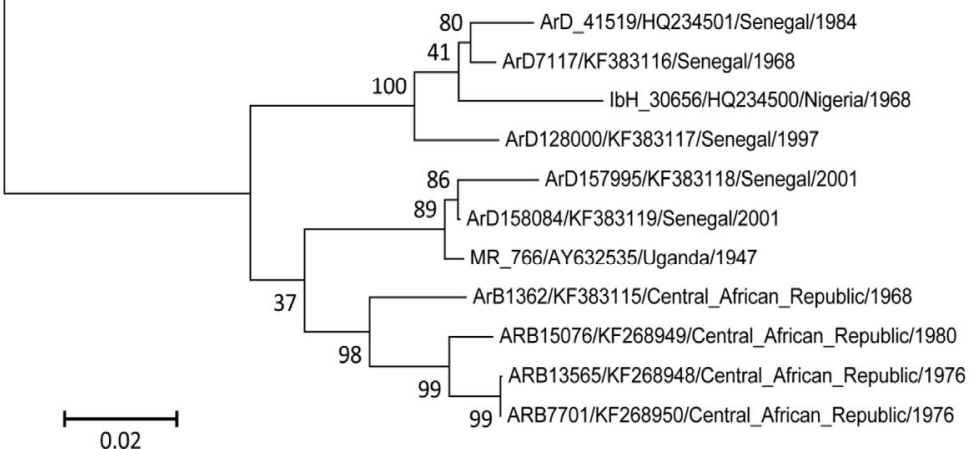

9月趾 PRVABC59/KU501215/Puerto_Rico/2015

89-Z1106033/KU312312/Suriname/2015

9. BeH818995/KU365777/Brazil/2015

BeH815744/KU365780/Brazil/2015

92. BeH819966/KU365779/Brazi//2015

SSABR 1/KU707826/Brazil/2015

99 . H/PF/2013/KJ776791/French_Polynesia/2013

L Sz01/China/2016

(1)

—_ H.sapiens-tc/THA/2014/SV0127-_14/KU681081/Thailand/2014

F 98 ZikaSPH2015/KU321639/Brazil/2015

43. Haiti/1225/2014/KU509998/Haiti/2014

- Natal_RGN/KU527068/Brazil/2015

103344/KU501216/Guatemala/2015

$1008375 / K U 501217 /$ Guatemala/2015

97 -MRS_OPY_Martinique_PaRi_2015/KU647676/Martinique/2015

89-Brazil-ZKV2015/KU497555/Brazil/2015

9\$ BeH819015/KU365778/Brazil/2015

Figure 1 The biological and genetic characterization of the ZIKV SZ01 strain. A, Isolation of SZ01 strain in the brain of suckling mice. Acute phase serum from the patient was injected intracranially into 2-day-old BALB/c mice. On the 7 days post inoculation, obvious nervous system symptoms were observed. B, Growth curves of the SZ01 strain in C6/36 cell culture. Monolayers of C6/36 cells were infected with the SZ01 viruses. At each time point, culture supernatant was collected and virus titer was determined using the qRT-PCR assays. C, Identification of the SZ01 strain by IFA. Vero cells were infected with SZ01 strain and 2 days after infection were fixed and incubated with the patient's convalescent phase serum. a, Specific reaction with convalescent phase serum from recovered ZIKV patient. b, Negative control. D, Plaque morphology of SZ01 strain on BHK-21 cells on the 3 days post inoculation. E, PCR products of the complete CDS of SZ01 strain. Lane 1-15 represents the different fragment amplified using 15 primer pairs, respectively. F, Phylogenetic analyses of ZIKV isolates based on the complete CDS using the Neighbor-Joining method by Tajima-Nei model. SZ01 strain was marked with red.

specific quantitative real time PCR (qRT-PCR) assays detected strong positive signal with a CT value of 22 in the brain tissue. The grinded supernatant was then inoculated into mosquito C6/36 cells and mammalian Vero cells, respectively, to further amplify the virus. Typical cytopathic effects were seen in both cells, and viral growth curve in C6/36 cells were determined using the qRT-PCR assays. The result showed that SZ01 strain replicated efficiently in C6/36 cells and peaked at 6 days post infection, with titers of $7.28 \times 10^{10}$ copies $\mathrm{mL}^{-1}$ (Figure 1B). Especially, standard plaque forming assay (Li et al., 2013) showed that SZ01 could produce typical small plaques in BHK-21 cells 3 days post inoculation (Figure 1D). The newly isolated ZIKV was further identified by indirect immunofluorescence assay (IFA) (Deng et al., 2011) using the convalescent phase serum from the second imported ZIKV patient (provided by the Guangzhou No.8 People's Hospital). The convalescent phase serum at 1:320 dilution could strongly react with the newly isolated SZ01 virus (Figure 1C).

Furthermore, to obtain the full genome of SZ01, a total of 15 primer pairs (Table S1) were used to generate the overlapping amplicons spanning the entire coding sequence (CDS) using the SuperScript III One-Step RT-PCR System (Life Technologies, USA). The resulting PCR products had an average length of 800 base pairs (Figure 1E). The PCR products were then sequenced by an ABI 3730 Sanger-based genetic analyzer and assembled using DNASTAR version 7.0. The complete CDS of SZ01 strain was submitted to GenBank under accession no. KU866423. The complete CDS of SZ01 strain is 10,272 nt in length, and the only open reading frame is predicted to encode a large polyprotein of 3,424 amino acids. Genome sequence alignment 
by MUSCLE in MEGA 6 demonstrates the SZ01 isolate has the highest similarity to the H/PF/2013 strain isolated in French Polynesia in 2013 (Cao-Lormeau et al., 2014). There are $99.4 \%$ homology in nucleotide and $99.7 \%$ in amino acid residues between the two strains. A total of eight amino acid variations were found in the envelope (E) protein, the nonstructural protein NS1, NS3 and NS5, respectively (Table S2). No additional amino acid mutation emerged in the conserved motifs of the predicted functional domains, including protease, helicase, RNA dependent RNA polymerase (RdRp), and methyl transferase (MTase) domain. It should be further determined whether these amino acid variations can impact the virulence of ZIKV in animals and viral fitness in mosquitoes.

To understand the origin of the Chinese isolate SZ01, all available complete CDS of ZIKV strains were retrieved from GenBank. Phylogenetic analysis based on the complete CDS was conducted by Neighbor-Joining method using MEGA version 6. As shown in Figure 1F, ZIKV strains fall into two lineages, the African and Asian lineage, and the SZ01 strain belongs to the Asian lineage. SZ01 strain was closely related to the H/PF/2013 strain. The patient had a travel history in Fiji and Samoa, and mosquito bites was reported during his stay in Samoa. Our phylogenetic results support the epidemiology evidence. Previously, ZIKV autochthonous transmission has been reported in these islands (Musso, 2015), and national health authorities should take special attention on the surveillance of the travellers from an area with an ongoing ZIKV outbreak.

Before this, no infectious ZIKV has been isolated in China ( $\mathrm{Li}$ et al., 2016). Here, we isolated the first ZIKV strain from a Chinese traveller returning to China. The success of ZIKV isolation in China will directly foster the development and evaluation of diagnosis kits, antiviral drugs and vaccines. Sequence alignment and phylogenetic analysis demonstrated that this Chinese isolate SZ01 belongs to the predominant Asian lineage. We also identified a panel of amino acid substitutions that deserves further investigation. Further studies are underway to clarify the pathogenesis and transmission efficacy using this ZIKV strain.
Compliance and ethics The author(s) declare that they have no conflict of interest.

Acknowledgements We thank the colleagues who help collect, deliver and prepare the clinical samples; and Dr. Fu-Chun Zhang (Guangzhou No.8 People's hospital) for providing the convalescent serum from recovered Zika patient. This work was supported by the State Key Laboratory of Pathogen and Biosecurity, China. Cheng-Feng Qin was supported by the Excellent Young Scientist Program from the National Natural Science Foundation of China (81522025) and the Newton Advanced Fellowship from the UK Academy of Medical Sciences and the National Natural Science Foundation of China (8151101191).

Cao-Lormeau, V.M., Roche, C., Teissier, A., Robin, E., Berry, A.L., Mallet, H.P., Sall, A.A., and Musso, D. (2014). Zika virus, French polynesia, South pacific, 2013. Emerg Infect Dis 20, 1085-1086.

Deng, Y.Q., Dai, J.X., Ji, G.H., Jiang, T., Wang, H.J., Yang, H.O., Tan, W.L., Liu, R., Yu, M., Ge, B.X., Zhu, Q.Y., Qin, E.D., Guo, Y.J., and Qin, C.F. (2011). A broadly flavivirus cross-neutralizing monoclonal antibody that recognizes a novel epitope within the fusion loop of $\mathrm{E}$ protein. PLoS One 6, e16059.

Duffy, M.R., Chen, T.H., Hancock, W.T., Powers, A.M., Kool, J.L., Lanciotti, R.S., Pretrick, M., Marfel, M., Holzbauer, S., Dubray, C., Guillaumot, L., Griggs, A., Bel, M., Lambert, A.J., Laven, J., Kosoy, O., Panella, A., Biggerstaff, B.J., Fischer, M., and Hayes, E.B. (2009). Zika virus outbreak on Yap Island, Federated States of Micronesia. N Engl J Med 360, 2536-2543.

Enfissi, A., Codrington, J., Roosblad, J., Kazanji, M., and Rousset, D. (2016). Zika virus genome from the Americas. Lancet 387, 227-228.

Fauci, A.S., and Morens, D.M. (2016). Zika Virus in the Americas--Yet Another Arbovirus Threat. N Engl J Med 374, 601-604.

Li, X.F., Deng, Y.Q., Yang, H.Q., Zhao, H., Jiang, T., Yu, X.D., Li, S.H., Ye, Q., Zhu, S.Y., Wang, H.J., Zhang, Y., Ma, J., Yu, Y.X., Liu, Z.Y., Li, Y.H., Qin, E.D., Shi, P.Y., and Qin, C.F. (2013). A chimeric dengue virus vaccine using Japanese encephalitis virus vaccine strain SA14-14-2 as backbone is immunogenic and protective against either parental virus in mice and nonhuman primates. J Virol 87, 13694-13705.

Li, X.F., Han, J.F., Shi, P.Y., and Qin, C.F. (2016). Zika virus: a new threat from mosquitoes. Sci China Life Sci 59, in press

Mlakar, J., Korva, M., Tul, N., Popovic, M., Poljsak-Prijatelj, M., Mraz, J., Kolenc, M., Resman Rus, K., Vesnaver Vipotnik, T., Fabjan Vodusek, V., Vizjak, A., Pizem, J., Petrovec, M., and Avsic Zupanc, T. (2016). Zika Virus Associated with Microcephaly. N Engl J Med 374, 951-958.

Musso, D. (2015). Zika Virus Transmission from French Polynesia to Brazil. Emerg Infect Dis 21, 1887

Open Access This article is distributed under the terms of the Creative Commons Attribution License which permits any use, distribution, and reproduction in any medium, provided the original author(s) and source are credited.

\title{
SUPPORTING INFORMATION
}

\author{
Table S1 Primers used for sequencing of ZIKV genome. \\ Table S2 The variation sites between the SZ01 strain and the H/PF/2013 strain of ZIKV.
}

The supporting information is available online at life.scichina.com and link.springer.com. The supporting materials are published as submitted, without typesetting or editing. The responsibility for scientific accuracy and content remains entirely with the authors. 